\title{
Device discovery for D2D communication in in-band cellular networks using sphere decoder like (SDL) algorithm
}

\author{
O. Hayat ${ }^{1,2^{*}}$ D, R. Ngah ${ }^{2}$ and Yasser Zahedi $^{3}$
}

\begin{abstract}
In the fifth generation (5G), it is anticipated that device-to-device (D2D) operation will be locally incorporated as a part without any bounds. In D2D network, multiple devices coexisting is a challenging subject of device discovery. The device discovery is performed under a visually impaired situation such as channel information, location, and the number of devices. In this paper, centralized device discovery is chosen due to power consumption and signaling overhead of the distributed system. A distinctive approach for device discovery in an in-band cellular network, based on the device's power, is suggested with an efficient technique which enhances the implementation of D2D communication and improves the accomplishment by alleviating the discovery issues. The group of devices forms a lattice structure, and it is positioned in the coverage area. The hypersphere is constructed based on the power knowledge of a discoverer device which helps for accurate and fast device discovery in a lattice structure. Besides, sphere decoder like (SDL) algorithm is applied for quick and precise discovery in the lattice structure. Simulation results present the performance of the proposed QR factorized lattice structure scheme regarding device power, enhanced in the number of discovered devices and controlled signaling overhead.
\end{abstract}

Keywords: Device-to-device (D2D) communication, Sphere decoder like (SDL) algorithm, Device discovery, Cellular network, End users (EU)

\section{Introduction}

Device-to-device (D2D) communication alludes to direct transmission between two devices without passing through the base station. It has been broadly anticipated to be an essential cornerstone to enhance system performance and bolster new amenities beyond 2020 in future fifth generation (5G) systems [1]. In 5G networks, it is anticipated that controlled D2D communication offers the open door for short-distance communication and local management and permits the isolation of local activity from the global activity, for example, local data offloading. D2D communication evacuates the data traffic heap load on the backhaul and center systems and decreases the vital exertion for managing data traffic at the center system. Due to proximity services, D2D

\footnotetext{
* Correspondence: ohayat@numl.edu.pk

${ }^{1}$ Department of Engineering, National University of Modern Language (NUML), H-9, Islamabad, Pakistan

${ }^{2}$ Wireless Communication Centre (WCC), Faculty of Electrical Engineering, Universiti Teknologi Malaysia, 81310 Johor Bahru, Malaysia Full list of author information is available at the end of the article
}

communication is viewed as a promising remedy for enhancing communication accomplishment and system capacity of long-term evolution-advanced (LTE-A) network. The potential enhancements in proximity services that can be given by D2D are not entirely exploited yet. In the $5 \mathrm{G}$ network, such confinement does not exist any longer, and it is anticipated that D2D operation in the in-band cellular network will be locally incorporated as a component without any bounds in the 5G network [2]. The in-band cellular network is considered in this research because interference and resource allocation is controlled by the base station or center system [3].

An important technique in deploying D2D communication is device discovery. Device discovery is characterized into two categories which are distributed discovery and centralized discovery. In the distributed discovery design, optimal resources and legitimate transmission power are apportioned with the consent of base station. However, this strategy involves complex signaling overhead and multifaceted nature of multiple transmitters to 
be composed [4]. In contrast, centralized device discovery design is adequately controlled by the base station and resources are managed by center system. Besides, the proximity services, for example, commercial announcement, public transport and municipality information, local programs, and impulsive social and corporate contacts infer decreased energy consumption and latency. Therefore, in D2D-enabled centralized networks, multiple devices coexisting is a challenging subject of device discovery for D2D communication to initiate the proximity services. However, latest reviews have concentrated on the D2D communication issues accepting that device discovery issue [5-7] is the vital problem and needs solution.

Discovery signal is designed in [8], minimizes collisions, and tries to improve the discovery process. A slight portion of resources used for device discovery and discovery signal which can be broadcasted with a minimum delay is proposed in $[9,10]$. Distinguishing between different discovery signals is difficult which leads to an increment in power consumption. Compressive sensing method has been suggested in [11], in which user detection decreases the collision for device discovery. However, when many devices are involved, active congestion will occur, and actual discovery will also be problematic. Device beaconing system is offered in [12]; it makes a discovery in the background of cellular traffic. If the devices are moving, then beaconing design for high-speed moving devices is much complicated. Neighbour discovery in LTE network, where the distributed orthogonal frequency multiple access (OFDMA) radio resources are used as user identities, is projected in [13], in which device discovery in high dense areas is not considered. Recommended technique in [14] uses the base station for discovery in which signaling flow from the base station can be used for discovery. In the large number of devices, the signaling overhead and discovery interference become problematic. Bioinspired and Firefly algorithm used for direct device discovery are advised in [15]. Due to the computational complexity, probability of misdetection is high. A device discovery scheme based on clustering has been introduced in [16] for a heterogeneous network, but the deficiency in the work is the sluggish discovery, and needs some techniques to decide clustering. All the previous discussions are guided to design a novel algorithm for device discovery which perform well in all aspects.

Therefore, in this article, the device's power-based sphere decipherer discovery scheme is proposed in which the device's power is incorporated for device discovery procedure. Power efficient, minimum collision, and low signaling overhead characterized discovery are achieved based on the utilization of a sphere decoder like (SDL) algorithm. The primary commitments of this article are encapsulated as follows:

- We propose a device's power-based device discovery to enhance the power efficiency and minimize the signaling overhead of typical D2D discovery.

- We propose disseminated collision fortitude algorithm and scheme which can take care of the D2D impairment like device power issue happening during the discovery phase.

- We evaluate the performance of the proposed algorithm by fusing the suppositions in recent LTE-A specialized reports [6].

The rest of the paper is organized as follows: Section 2 provides the overview of discovery resources which are used in discovery procedure, while Section 3 consists of the D2D discovery system model. In Section 4, power scheme for device discovery is elaborated, and Section 5 contains the results' analysis. In the end, the paper is concluded in Section 6.

\section{Resources for device discovery}

In general, devices in D2D communication may misuse both downlink and uplink resources for cellular communication [2]. In this work, we expect that device discovery uses uplink resources based on reusing the uplink transmission chain [17] Therefore, to ensure the excellent performance of device discovery, shared radio resources are suggested alternately with dedicated radio resources. Accordingly, D2D devices, taking an interest in discovery, will choose one radio resource blocks (RRB) among the intermittent discovery radio resources. A case of discovery period and RRB is exhibited in Fig. 1. The D2D devices can transmit own discovery signal on their chosen RRB one time and tune for reception of discovery signals from other D2D devices. During the discovery period, each D2D device takes an interest in the discovery procedure only, and other sorts of communications are not permitted.

Each device surveys all RRB's received power level and selects the RRB which has the most reduced power level [18]. Appropriately, numerous devices situated far away may pick a similar resource. On the other hand, every device randomly chooses RRB resources for discovery signal transmission. We concentrate on a random choice due to the human mobility pattern [19]. There are two scenarios for device discovery that depend on mobility, haphazard walk scenarios, and velocity scenarios in which discovery is computed. Haphazard walk model does not much depend on environment changes, while mobility depends on context and velocity, which might be unknown or partially known or measurable by some models [20]. The sensing-based determination is wasteful 


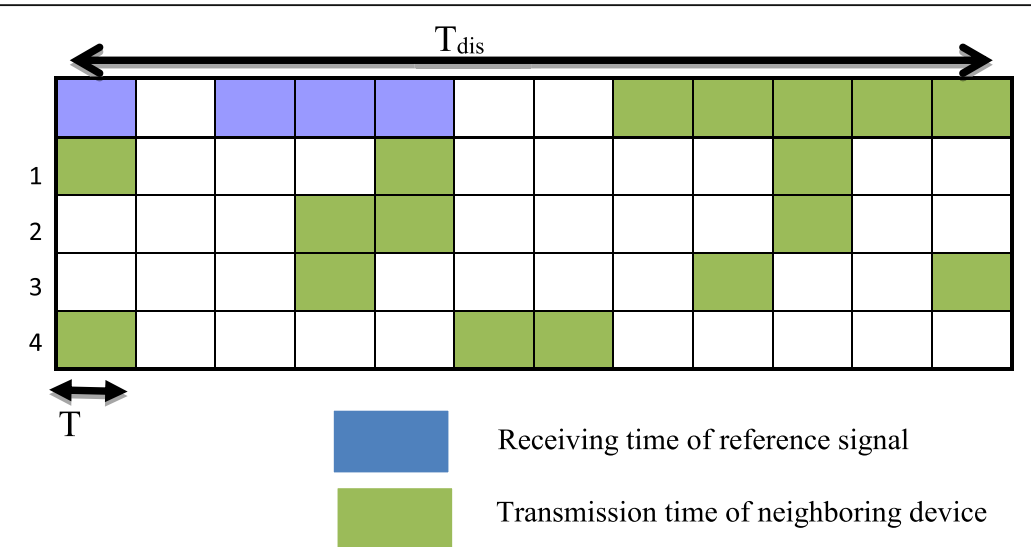

Fig. 1 RRB for discovery $T_{\text {dis }}$ is the total time for discovery and $T$ is the distributed time for each device. Legend: receiving time of reference signal and transmission time of neighboring devices

when the sensing outcomes are obsolete rapidly, for example, under high-mobility situation. When two or more devices reuse similar discovery resources in the vicinity, a collision may happen because of the asynchronous transmission [21]. Accordingly, these neighboring D2D devices can neither distinguish each other nor be recognized because of the mutual interference.

\section{D2D device discovery system model}

In this section, we present a short preface for the D2D device discovery system model. A system model for device discovery where $R$ is the radius of a sphere made by the discoverer device is shown in Fig. 2. In our analysis, we confine our extension of synchronous D2D device discovery, for example, all D2D devices are in time synchronization and coverage area reference can be acquired from the base station downlink transmission. This devours significantly less energy and discovery time compared with asynchronous. For synchronous device discovery, each D2D device can be dynamic within a

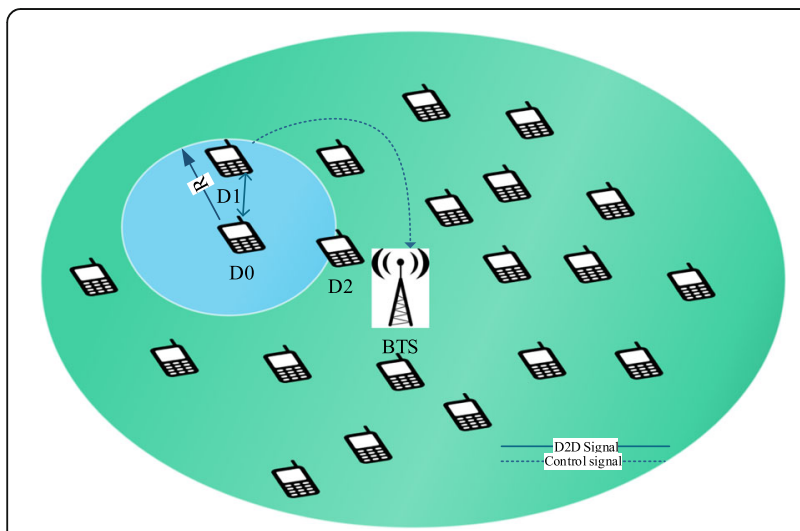

Fig. 2 System model for device discovery where $R$ is the radius of a sphere made by the discoverer device. Legend: D2D signal and control signal predefined discovery time, which shows up intermittently, such as D2D device occasionally awakes to accomplish the discovery process utilizing the D2D RRB. After finishing the periodic discovery, D2D devices start dozing until subsequent discovery period starts. When a D2D device has discovered a fancied target D2D device by accepting a signal, it can build up a D2D interface for direct communication. In cellular network topology, the transmission of mobile devices is relied upon base station.

Appropriately, if various cellular devices have a place with various cells, reuse a common resource in a cell edge, a cellular device signal interferes with the neighboring base station. Then again, in D2D systems, there coexist numerous D2D devices which can be both receiver and transmitter. Under this topology, radiated signals from various transmitting D2D devices will reach proximal D2D device's receiver. Note that various receivers are possibly presented to endure high interference by numerous D2D links. Accepting the quantity of $k \mathrm{D} 2 \mathrm{D}$ devices in D2D systems, the greatest number of D2D links is $k(k-1)$, which has a polynomial ratio [18].

\section{Power scheme for discovery}

In the LTE-A, D2D users and cellular users are multiplexed at the same uplink channel orthogonal frequency division multiplexing (OFDM) physical RRB. The received signal from the $k$ cellular devices or D2D devices can be modelled as [22]

$$
\begin{gathered}
y_{k}=\alpha_{k, k} \boldsymbol{H}_{k, k} \boldsymbol{T}_{k} x_{k}+\sum_{j \neq k} \alpha_{k, j} \boldsymbol{H}_{k, j} \boldsymbol{T}_{j} x_{j}+n_{k} \\
\alpha_{k, j}=\sqrt{P_{j} d_{k, j}^{-\rho} X_{k, j} / N_{t}} \text { is a scalar depending upon the }
\end{gathered}
$$

total transmitting power $P_{j}$ for $j$ user, $\chi_{k, j}$ is shadowing fading, and $d_{k, j}$ is the distance between $k$ receivers and $j$ transmitters with path loss $\rho . x_{k} \in \mathbb{C}^{N_{t} \times 1}$ normalized data vector with zero 
mean and uncorrelated $\mathrm{E}\left(x_{k} x_{k}^{\dagger}\right)=I_{N_{t}} . \boldsymbol{H}_{k, j}$ implies $\left(N_{r} \times N_{t}\right)$ channel matrix with $N_{r}$ which receive antennas and $N_{t}$ which transmit antennas, and $\boldsymbol{T}_{k}$ refers to $k$ th user's diagonal power matrix. To keep the total power constant, $\boldsymbol{T}_{k}$ must satisfy the following condition:

$$
\operatorname{trace}\left(\boldsymbol{T}_{k} \boldsymbol{T}_{k}^{\dagger}\right)=\sum_{i=1}^{N_{t}}\left|\boldsymbol{T}_{k}^{(i, i)}\right|^{2}=N_{t} \quad \forall \quad k
$$

$n_{k}$ is additive white Gaussian noise (AWGN) with zero mean and covariance is

$$
\boldsymbol{R}_{n_{k}}=\mathrm{E}\left(\mathrm{n}_{\mathrm{k}} \mathrm{n}_{\mathrm{k}}^{\dagger}\right)=\sigma_{n}^{2} \boldsymbol{I}_{\boldsymbol{N}_{r}} \quad \forall \quad \mathrm{k}
$$

Signal model can be written as

$$
y_{k}=\alpha_{k, k} \boldsymbol{H}_{k, k} \boldsymbol{T}_{k} x_{k}+\boldsymbol{z}_{k}+n_{k}
$$

$\boldsymbol{z}_{k}=\sum_{j \neq k} \alpha_{k, j} \boldsymbol{H}_{k, j} \boldsymbol{T}_{j} x_{j}$ is the interference matrix for D2D and cellular users with covariance

$$
\boldsymbol{R}_{z_{k}}=\mathrm{E}\left[\mathbf{z}_{\mathbf{k}} \mathbf{z}_{\mathrm{k}}^{\dagger}\right]=\sum_{j \neq k} \alpha_{k, j}^{2} \boldsymbol{H}_{k, j} \boldsymbol{T}_{j} \boldsymbol{T}_{j}^{\dagger} \boldsymbol{H}_{k, j}^{\dagger}
$$

The power of the $j$ device is

$$
\boldsymbol{P}_{j}=\frac{\boldsymbol{P}_{t} \boldsymbol{H}_{t, j}}{\gamma_{r}^{d} \sum_{j \neq k} \boldsymbol{H}_{k, j}}-\frac{\sigma_{n}^{2}}{\sum_{j \neq k} \boldsymbol{H}_{t, j}}
$$

$\boldsymbol{H}_{t, j}$ is the channel response between a base station and $j$ device. $\boldsymbol{H}_{k, j}$ are channel responses between $k$ devices and $j$ devices. $\gamma_{r}^{d}$ is the discovery signal to interference noise ratio (SINR) at the receiver device. Successful device discovery is only achieved when SINR of decoding signal is greater than the threshold value of SINR $\gamma_{r}^{\text {th }}$; therefore,

$$
\boldsymbol{P}_{t} \geq \gamma_{r}^{t}\left(\sum_{j \neq k} \frac{\boldsymbol{P}_{J} \boldsymbol{H}_{k, j}}{\boldsymbol{H}_{t, j}}+\frac{\sigma_{n}^{2}}{\boldsymbol{H}_{t, j}}\right)
$$

From (7), $\boldsymbol{P}_{j} \geq \gamma_{r}^{\text {th }}\left(\frac{1}{\beta \boldsymbol{P}_{t}-\sigma_{n}^{2}}\right)$, where $\beta$ is the ratio of $\frac{\boldsymbol{H}_{t, j}}{\boldsymbol{H}_{k, j}}$

$$
\delta_{t}= \begin{cases}0 & \gamma_{r}^{d}<\gamma^{t h} \\ 1 & \gamma_{r}^{d} \geq \gamma^{t h}\end{cases}
$$

where $\delta_{t} \in\{0,1\}$. If $\delta_{t}=0$ no discovery, $\delta_{t}=1$ device has been discovered and $\gamma_{r}^{\text {th }}=\frac{s}{I+n}$ in which $s, I$, and $n$ are the signal power, interference, and noise power respectively. From (1)

$$
\mathrm{y}_{\mathrm{k}}=\mathbf{H}_{k, j} x_{k}+\mathrm{n}_{k}
$$

By taking the QR decomposition of $\mathbf{H}_{k, j}$ from (9)

$$
\begin{gathered}
\mathbf{R}=\left[\begin{array}{ccc}
r_{11} & r_{12} \cdots & r_{1 j} \\
0 & r_{22} \cdots & r_{2 j} \\
\vdots & \ddots & \vdots \\
0 & \cdots & r_{j j}
\end{array}\right] \\
\mathbf{Q}^{\mathrm{H}} \mathbf{y}_{\mathbf{k}}=\mathbf{R} \mathbf{x}_{\mathbf{k}}+\mathbf{Q}^{\mathrm{H}} \mathrm{n}_{k}
\end{gathered}
$$

$$
\left[\begin{array}{c}
y_{1} \\
y_{2} \\
\vdots \\
y_{k}
\end{array}\right]=\left[\begin{array}{ccc}
r_{11} & r_{12} \cdots & r_{1 j} \\
0 & r_{22} \cdots & r_{2 j} \\
\vdots & \ddots & \vdots \\
0 & \cdots & r_{j j}
\end{array}\right]\left[\begin{array}{c}
x_{1} \\
x_{2} \\
\vdots \\
x_{k}
\end{array}\right]+\mathbf{Q}^{\mathrm{H}} \mathrm{n}_{\mathrm{k}}
$$

The SDL algorithm can reduce the complexity by searching for the closest device among the possible lattice devices that lie within a hypersphere of radius $R$ around the discoverer device $x$. Mathematically, the SDL algorithm solves the problem as:

$$
\hat{x}=\arg \underset{\mathrm{s}_{i} \in \mathcal{O}^{M_{j}}:\|\mathrm{y}-\mathbf{H s}\|^{2} \leq R^{2}}{ }\|\mathrm{y}-\mathbf{H x}\|^{2}
$$

where $\mathbf{H} \in \mathcal{R}^{m \times n}, \quad x \in \mathcal{R}^{n \times 1}$, and $\mathcal{O}^{N_{T}}$ show the $m$-dimensional lattice points. $\mathcal{O}$ shows the device's constellation. The SDL algorithm only searches for the maximum likelihood devices from among the lattice devices that lie within a hypersphere of radius $R$ around the discovery received signal as presented in Fig. 3.

Calculating the distance between each lattice device to the discoverer reduces the exhaustive search. The SDL can cleverly find the set of lattice devices within the hypersphere. This is the simple set of lattice devices in an interval around the discoverer. Similarly, if we know the set of lattice devices in a $k$-dimensional space that lies within a hypersphere, then the possible values of the $(k+1)$ th coordinate for the set of lattice devices [23]. We can thus recursively find and check all lattice devices in the hypersphere. Mathematically, it can be expressed as:

$$
\mathbf{H}=\mathbf{Q}\left[\begin{array}{c}
\mathbf{R}_{N_{j} \times N_{j}} \\
\mathbf{0}\left(N_{k}-N_{j}\right) \times N_{j}
\end{array}\right]
$$

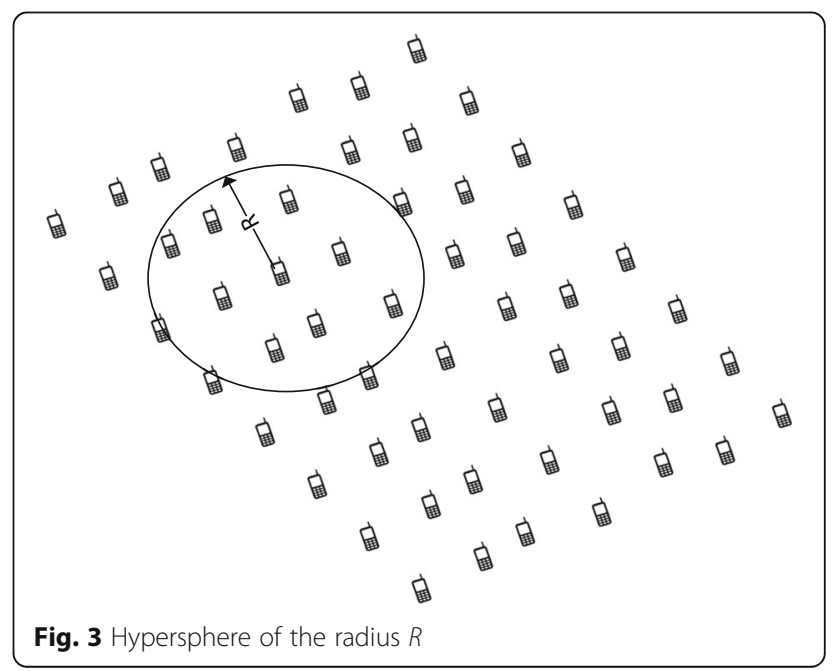


where the unitary matrix is partitioned as $\mathbf{Q}=\left[\begin{array}{ll}\mathbf{Q}_{\mathbf{1}} & \mathbf{Q}_{\mathbf{2}}\end{array}\right]$ such that $\mathbf{Q}_{\mathbf{1}}$ contains the first $N_{T}$ columns of $\mathbf{Q}$. With a little manipulation, it can be shown that the hypersphere constraint becomes

$$
\mathbf{R}^{2} \geq\|\underbrace{\mathbf{Q}_{1}^{H} \mathbf{y}}_{\mathbf{x}}-\mathbf{R x}\|^{2}
$$

It also can be written as

$$
\mathbf{R}^{2} \geq \sum_{K=1}^{M_{j}}\left(\mathrm{y}_{K}-\sum_{l=K}^{M_{j}} \mathrm{r}_{K, l} \mathrm{x}_{l}\right)^{2}
$$

where $\mathrm{y}_{K}, \mathrm{x}_{K}$, are the $k$ th element of $y$ and $x$. Also $\mathrm{r}_{k, l}$ is the $(k, l)$ th element of the upper triangular matrix $\mathbf{R}$. To satisfy the condition in (14), a necessary but not sufficient condition is

$$
\mathbf{R}^{2} \geq\left(\mathrm{y}_{N_{j}-r_{N_{j}}, N_{j} x_{N_{j}}}\right)^{2}
$$

This implies the following condition on $\mathrm{x}_{N_{j}}$

$$
\left\lceil\frac{\mathrm{y}_{N_{j}}-\boldsymbol{R}}{r_{N_{j}, N_{j}}}\right\rceil \leq x_{N_{j}} \leq\left\lfloor\frac{\mathrm{y}_{N_{j}}+\boldsymbol{R}}{r_{N_{j}, N_{j}}}\right\rfloor
$$

Likewise, if we define $\mathrm{y}_{\left(M_{T}-1 \mid m_{T}\right)}=\mathrm{y}_{M_{T}-1}-r_{M_{T}-1, M_{T}} \mathrm{~s}_{M_{T}}$, we get the following necessary condition on $\mathrm{s}_{M_{T}-1}$

$$
\left\lceil\frac{\mathrm{y}_{\left(N_{j}-1 \mid n_{j}\right)}-\boldsymbol{R}_{N_{j}-1}}{r_{N_{j}-1, N_{j}-1}}\right\rceil \leq \mathrm{x}_{M_{j}} \leq\left\lfloor\frac{\mathrm{y}_{\left(N_{j}-1 \mid n_{j}\right)}+\boldsymbol{R}_{N_{j}-1}}{r_{N_{j}-1, N_{j}-1}}\right\rfloor
$$

The SDL algorithm proceeds similarly to obtain the lattice devices within the hypersphere.

\section{Result analysis}

A simulation setup model [24] is established to verify the proposed discovery scheme. A centralized model with the discovery area $500 \times 500 \mathrm{~m}$ and 50 devices are deployed randomly using Poisson point process (PPP) as shown in Fig. 4. Dotted lines are connected devices, qualified for direct D2D communication and relay communication. RRB selection is performed based on received power of discovery resources. These devices send discovery signal through a base station on given RRB to discover the neighbor devices. RRBs are not chosen with the lowest received power which causes to reduce the probability of discovery of devices. If the required device is to be found in its proximal using the condition (8), then the base station will allow for D2D communication. Using power conditions, some devices may make D2D LAN via relay devices under the control link of the base station.

Discovery ratio depends upon the searching power of each device that lies in the cellular network area as

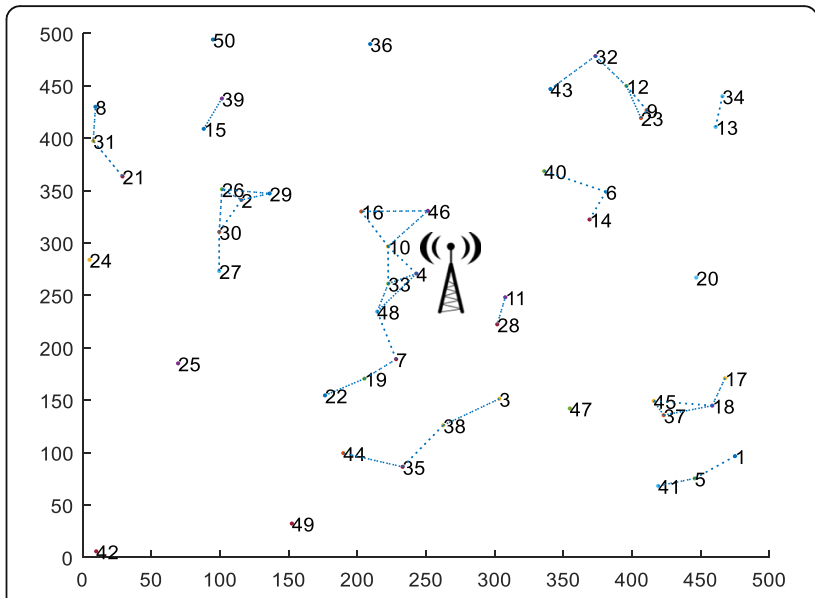

Fig. 4 Randomly deployed devices in a cellular network in $500 \times 500 \mathrm{~m}$ area

expressed in (6). Devices in the cell area make lattice structure, and each device from the lattice has a radius $R$. By the SDL algorithm, the distance of each device from the discoverer is calculated in lattice devices. $Q \boldsymbol{R}$ factorization helps to minimize the searching power as expressed in (18) to find out the proximal devices. Performance of proposed $\boldsymbol{Q R}$ factorized lattice structure base scheme is shown in Fig. 5, and the results are compared with those of the Gaussian method. In the ideal situation, minimum discovery cycles discover maximum devices. In our proposed model, discovery ratio meets the ideal. At five discovery signals, near about 1000 devices have been discovered.

Further, we applied our proposed scheme on centralized and distributed system and compared the results. Centralized and distributed scheme for device discovery

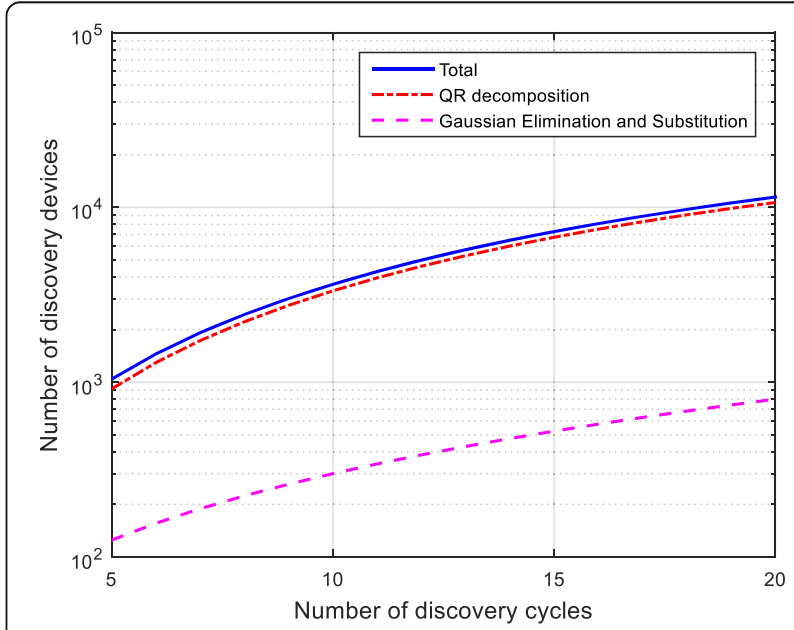

Fig. 5 Performance of proposed QR factorized lattice structure base scheme. Legend: total result, QR decomposition, and Gaussian elimination and substitution 


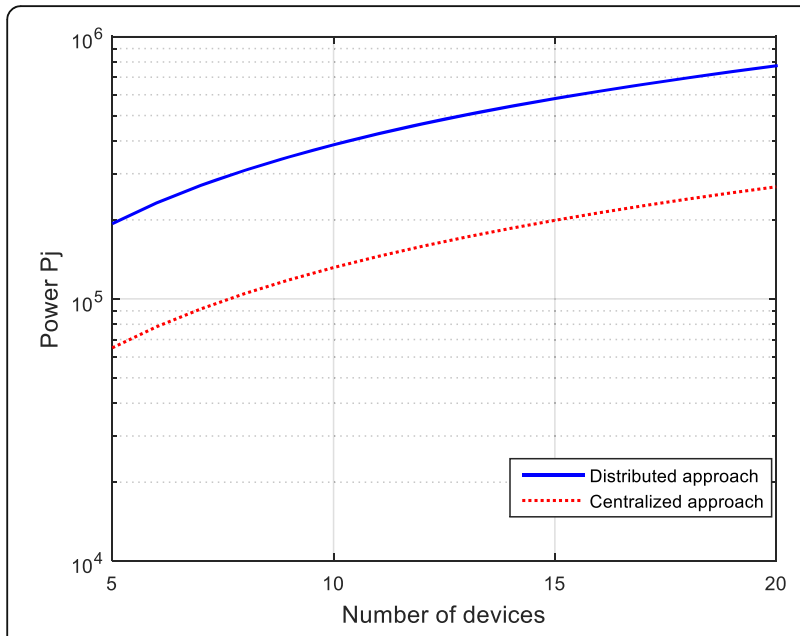

Fig. 6 Power consumption in $j$ devices while power Pj scale is in nano. Legend: distributed approach and centralized approach

has been discussed in the Section 1. In the distributed plan, the main issue is power consumption and signaling overhead. So, in this proposed model, these problems have been overcome as shown in Fig. 6 and 7 respectively. Centralized approache is better in-term of power consumption and signaling overhead respectively. In the in-band cellular network device discovery, a centralized approach $0.1 \mathrm{mw}$ power is consumed on discovery signal when 15 devices have been discovered.

When the SDL algorithm with $\boldsymbol{Q R}$ factorization is applied, the signaling overhead significantly decreases as shown in Fig. 8. Fast device discovery depends upon the number of transmitted discovery signal. If the discovery signal is significant, then signaling overhead occurs. In our proposed method in a centralized approach, signaling overhead decreases. Initially, five devices to discover

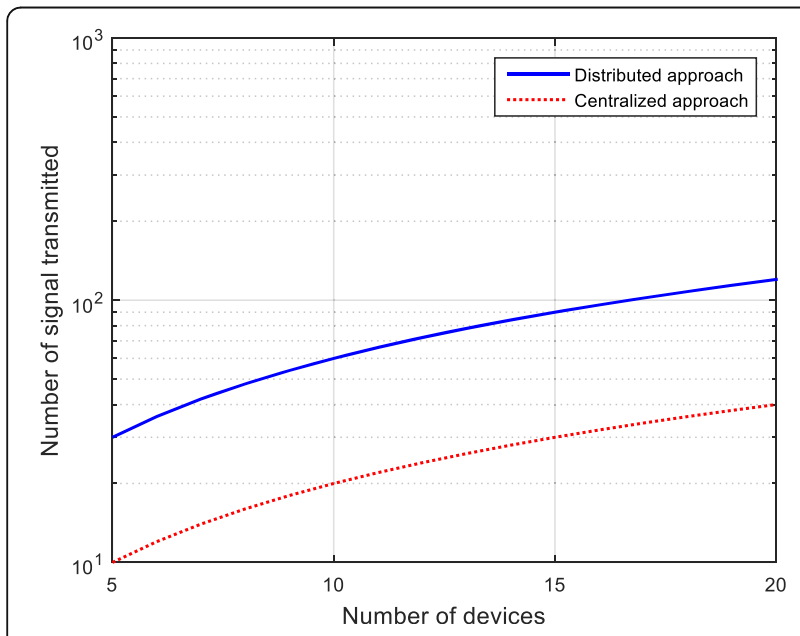

Fig. 7 Signaling overhead for distributed and centralized approaches. Legend: distributed approach and centralized approach

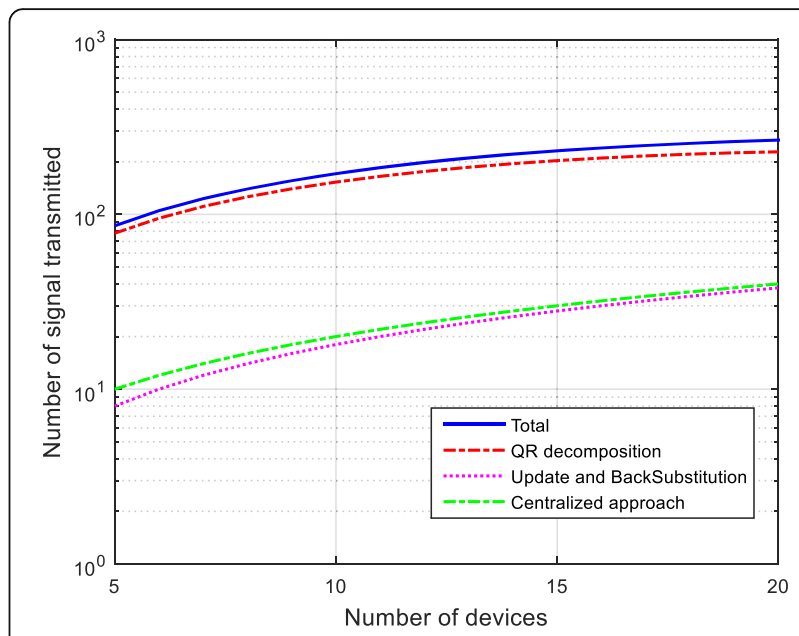

Fig. 8 Signaling overhead comparison of different schemes with proposed scheme. Legend: total result, QR decomposition, update and back substitution, and centralized approach

the proximal users need 10 signals, and 10 devices need 20 signals; therefore, signaling overhead decrease in a centralized approach.

\section{Conclusions}

D2D communication isolates the local cellular traffic to the global cellular traffic. Accordingly, device discovery is an underlying strategy in D2D communication. In this research, device's power-based discovery method is proposed in the centralized cellular network. This clear approach for device discovery enhances the accomplishment by alleviating the discovery issues as fast discovery, minimum signaling overhead, and power consumption. Our proposed methodology is the $\boldsymbol{Q R}$ factorized lattice structure of devices in a specific area. Discoverer device creates a hypersphere around it and sends a discovery signal in the hypersphere using sphere decoder like an algorithm. Discoverer device visits all the lattice devices that lie in the hypersphere and finds the closest one to initiate the D2D communication. Using the hypersphere, the designed discovery scheme saves the discovery time and power for searching of unwanted devices. In future, this plan can be implemented on a distributed system and can spare the time by exhaustive search.

\section{Acknowledgements}

The authors would like to express their gratitude to the Ministry of Higher Education (MOHE) in Malaysia and Universiti Teknologi Malaysia (UTM) for providing the financial support for this research through the HICOE grant (R. J130000.7823.4J215). The grant is managed by Research Management Center (RMC) at UTM.

\section{Funding}

The Ministry of Higher Education (MOHE) in Malaysia and the Universiti Teknologi Malaysia (UTM) are providing the financial support for this research through the HICOE grant. 


\section{Availability of data and materials}

Mostly, I got the writing material from different journals as presented in the references. A MATLAB tool has been used to simulate my concept.

\section{Authors' contributions}

$\mathrm{OH}$, an assistant professor at NUML, H-9, Islamabad, and a student of PhD in Wireless Communication Center (WCC), Faculty of Electrical Engineering, UTM Johor Baharu, Malaysia under the supervision of associate professor RN, implemented the idea regarding device discovery. $Y Z$, assistant professor at Department of Information and Communication Engineering, Basrah University College of Science and Technology, Basrah, Iraq, helped to implement and write this paper. Overall, this paper is built under the approval and supervision of RN. All authors read and approved the final manuscript.

\section{Competing interests}

The authors declare that they have no competing interests.

\section{Publisher's Note}

Springer Nature remains neutral with regard to jurisdictional claims in published maps and institutional affiliations.

\section{Author details}

${ }^{1}$ Department of Engineering, National University of Modern Language (NUML), H-9, Islamabad, Pakistan. 'Wireless Communication Centre (WCC), Faculty of Electrical Engineering, Universiti Teknologi Malaysia, 81310 Johor Bahru, Malaysia. ${ }^{3}$ Department of Information and Communication Engineering, Basrah University College of Science and Technology Basrah, Iraq.

Received: 11 September 2017 Accepted: 18 March 2018

Published online: 03 April 2018

\section{References}

1. M Agiwal, A Roy, N Saxena, "Next generation 56 wireless networks: A comprehensive survey," IEEE Communications Surveys \& Tutorials. 18(3), 1617-1655 (2016)

2. O Hayat, R Ngah, Y Zahedi, "Cooperative Device-to-Device Discovery Model for Multiuser and OFDMA Network Base Neighbour Discovery in In-Band $5 \mathrm{G}$ Cellular Networks," Wireless Personal Communications. 97(3) 4681-4695 (2017)

3. M Dohler, $\mathrm{T}$ Nakamura, $5 \mathrm{G}$ mobile and wireless communications technology. Cambridge University Press, University Printing House, Cambridge, (2016)

4. G Fodor, S Roger, N Rajatheva, SB Slimane, T Svensson, P Popovski, et al., An overview of device-to-device communications technology components in METIS. IEEE Access 4, 3288-3299 (2016)

5. K Sharmila, V Mohan, C Ramesh, SP Munda, in Advances in Electrical, Electronics, Information, Communication and Bio-Informatics (AEEICB), 2016 2nd International Conference on. Proximity services based device-to-device framework design for direct discovery (2016), pp. 499-502

6. D Tsolkas, N Passas, L Merakos, Device discovery in LTE networks: a radio access perspective. Comput. Netw. 106, 245-259 (2016)

7. Z Wu, VD Park, J Li, Enabling device to device broadcast for LTE cellular networks. IEEE Journal on Selected Areas in Communications 34, 58-70 (2016)

8. S-Y Lien, C-C Chien, F-M Tseng, T-C Ho, 3GPP device-to-device communications for BeyonD 4G cellular networks. IEEE Commun. Mag. 54, 29-35 (2016)

9. Qualcomm Technologies.Inc, "LTE Direct Always-on Device-to-Device Proximal Discovery," Qualcomm Technologies, 5775 Morehouse Drive San Diego, CA 92121 U.S.A. (2014)

10. S Park, S Choi, in 2014 IEEE Global Communications Conference. Expediting D2D discovery by using temporary discovery resource (2014), pp. 4839-4844

11. L Zhang, J Luo, D Guo, Neighbor discovery for wireless networks via compressed sensing. Perform. Eval. 70, 457-471 (2013)

12. K Doppler, CB Ribeiro, J Kneckt, in Wireless Communication, Vehicular Technology, Information Theory and Aerospace \& Electronic Systems Technology (Wireless VITAE), 2011 2nd International Conference on. Advances in D2D communications: energy efficient service and device discovery radio (2011), pp. 1-6

13. F Baccelli, N Khude, R Laroia, J Li, T Richardson, S Shakkottai, et al., in 2012 Fourth International Conference on Communication Systems and Networks (COMSNETS 2012). On the design of device-to-device autonomous discovery (2012), pp. 1-9
14. H Tang, Z Ding, BC Levy, Enabling D2D communications through neighbor discovery in LTE cellular networks. IEEE Trans. Signal Process. 62, 5157-5170 (2014)

15. S-L Chao, H-Y Lee, C-C Chou, H-Y Wei, Bio-inspired proximity discovery and synchronization for D2D communications. IEEE Commun. Lett. 17, 23002303 (2013)

16. D Xenakis, L Merakos, M Kountouris, N Passas, C Verikoukis, Distance distributions and proximity estimation given knowledge of the heterogeneous network layout. IEEE Trans. Wirel. Commun. 14, 5498-5512 (2015)

17. CJ Katila, C Buratti, MD Abrignani, R Verdone, Neighbors-aware proportional fair scheduling for future wireless networks with mixed MAC protocols. EURASIP J. Wirel. Commun. Netw. 2017, 93 (2017)

18. J Hong, S Park, S Choi, "Novel power control and collision resolution schemes for device-to-device discovery," Peer-to-Peer networking and applications, 9(5) 913-922 (2016)

19. Y Liu, C Liu, NJ Yuan, L Duan, Y Fu, H Xiong, et al., Intelligent bus routing with heterogeneous human mobility patterns. Knowl. Inf. Syst. 50, 383-415 (2017)

20. AH Sayed, A Tarighat, N Khajehnouri, Network-based wireless location: challenges faced in developing techniques for accurate wireless location information. IEEE Signal Process. Mag. 22, 24-40 (2005)

21. M Klügel, W. Kellerer, "Dominant factors for device-to-device occurrence probabilities in cellular networks," Wireless Networks, 1-13 (2017)

22. H Sun, M Sheng, X Wang, Y Zhang, Y Shi, Distributed cooperative device-todevice transmissions underlaying cellular networks. Wirel. Netw 21, 1411-1423 (2015)

23. O Hayat, M Qaisrani, M Akbar, in Emerging Technologies (ICET), 2010 6th International Conference on. Sphere decoding in the presence of channel uncertainty, 216-220 (2010)

24 N. Papalexidis, "Distributed Algorithms for Beamforming in Wireless Sensor Networks," DTIC Document, Naval Postgraduate School Monterey, CA, June 2007

\section{Submit your manuscript to a SpringerOpen ${ }^{\circ}$ journal and benefit from:}

- Convenient online submission

- Rigorous peer review

- Open access: articles freely available online

- High visibility within the field

- Retaining the copyright to your article

Submit your next manuscript at $\boldsymbol{\nabla}$ springeropen.com 